Indexaciones: Repositorio de Revistas UCR, DIALNET, Latindex, REDALYC Directorio y recolector de recursos digitales del Ministerio de Cultura de España, Directory of Open Access Journals. Diálogos Revista Electrónica de Historia ISSN 1409- 469X. Número especial 2008. Dirección web: http://historia.fcs.ucr.ac.cr/dialogos.htm

\title{
El desarrollo de la agricultura en la Región Pacífico Central de Costa Rica (1909-1955)
}

\author{
Jorge Bartels Villanueva
}

Máster en Economía y coordinador de Docencia, Sede del Pacífico, Universidad de Costa Rica. Correo electrónico: jorge.bartels@ucr.ac.cr

\section{Ricardo Martínez Esquivel}

Bachiller en Historia y estudiante de maestría académica en Historia de la Universidad de Costa Rica. Correo electrónico: shoremricardo@yahoo.es 


\section{Introducción}

La presente investigación analiza el desenvolvimiento de la actividad agrícola entre los años de 1909 a 1955 en la Región Pacífico Central de Costa Rica. La fuente primaria utilizada en este trabajo son los censos agrícolas de 1909, 1914, del periodo 1923-1927 y el de 1955; siendo el primero y el último censo, los que delimitan el periodo en estudio. Con respecto al periodo de los años 1923 a 1927, hemos decidido realizar un promedio de estos, lo cual vendría a menguar la dificultad que presentan algunos de estos censos al no poseer los datos completos.

La Región Pacífico Central de Costa Rica la constituyen los cantones de la provincia de Puntarenas: Puntarenas, Esparza, Montes de Oro, Garabito y Parrita; y los cantones de la provincia de Alajuela: San Mateo y Orotina. Por otra parte, vale señalar que estos cantones no siempre existieron como tales o incluso del todo, por lo que en la primera parte de esta investigación explicaremos la evolución histórica de la región. En una segunda parte de este trabajo analizaremos los cambios y permanencias en la explotación agrícola del suelo en la región, relacionando dicho proceso con el acontecer del mercado interno del país y la participación de Costa Rica en el mercado internacional.

\section{La Región Pacífico Central de Costa Rica}

La idea del Estado de dividir Costa Rica en regiones diferentes a las provincias fue con el propósito de una mejor administración y planificación del país. Comprendemos por región al "resultado de una forma específica de desarrollo económico, social y político, basado en diversas relaciones y procesos históricos llevados a cabo por grupos sociales sobre un territorio determinado". ${ }^{1}$ Con la regionalización del país se ha pretendido ayudar a las zonas menos desarrolladas de éste. El Estado costarricense ha buscado facilitar procesos de planificación, coordinación y ejecución de proyectos en las distintas comunidades. ${ }^{2}$

La primera propuesta de regionalización de Costa Rica fue elaborada por el geógrafo alemán Helmunt Nuhn, ${ }^{3}$ quien 1972 realizó su planteamiento en base a un estudio de estructuras

$1 \quad$ Ronulfo Alvarado Salas, "Regiones y cantones de Costa Rica", Serie cantones de Costa Rica 2, Instituto de Fomento y Asesoría Municipal (IFAM), Dirección de Gestión Municipal, Sección de Investigación y Desarrollo, San José, 2003, 7

$2 \quad$ Ibid., 3

3 Helmut Nuhn, Regionalización de Costa Rica para la planificación y el desarrollo, San José: Oficina de Planificación, 1973 
geográficas según criterios geofísicos y socioeconómicos. Nuhn identificó seis regiones: Región Central, Región Pacífico Norte, Región Pacífico Central, Región Pacífico Sur, Región Norte y Región Atlántica, las cuales se aplican al país en el gobierno de José Figueres (1970-1974). Posteriormente en el gobierno de Rodrigo Carazo (1978-1982) se simplifican en cinco: Central, Chorotega, Brunca, Huetar Atlántica y Huetar Norte. En el esquema anterior la región Central absorbió la del Pacífico Central.

En el gobierno de Luis Alberto Monge (1982-1986) se recupera la región del Pacífico Central. En el gobierno Calderón Fournier (1990-1994) son establecidas las provincias de Heredia y Cartago como regiones, esto argumentándose que el esquema de Nuhn era insuficiente porque sólo consideró la situación geográfica y económica de la década de 1970. ${ }^{4}$ Este último cambio no fructificó y se dejó de lado la regionalización del país hasta el gobierno de Abel Pacheco (2002-2006) donde se retoma con la división establecida durante la administración de Monge.

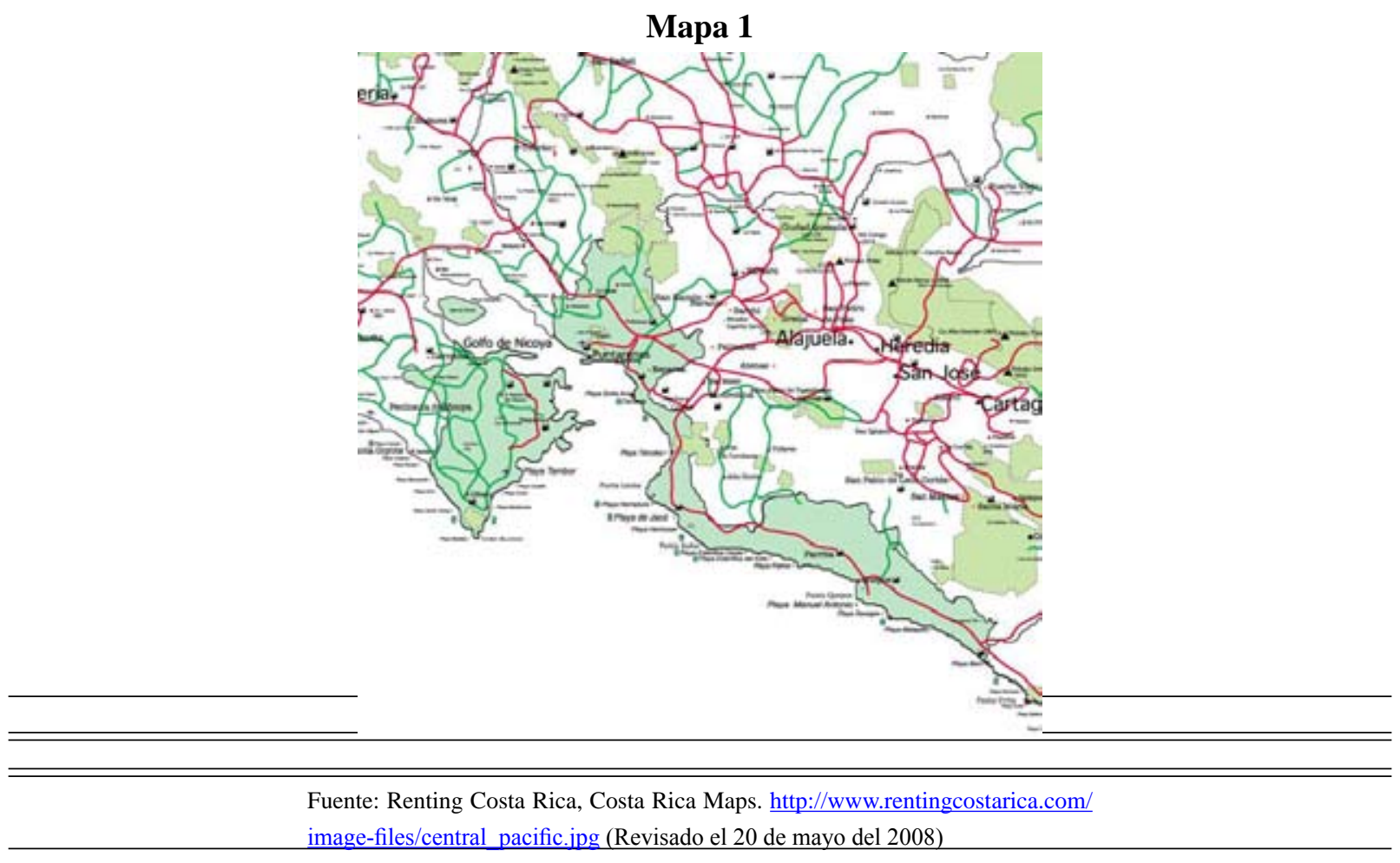

4

Alvarado, "Regiones y cantones de Costa Rica", 5-6 
En el Mapa 1 observamos como la Región Pacífico Central limita al norte con los cantones de Puriscal, Tarrazú. Aserrí, Acosta y Turrubares de la provincia de San José; el cantón de San Ramón de la provincia de Alajuela; y el cantón Abangares de la provincia de Guanacaste. Al este con los cantones de Turrubares, Dota y Pérez Zeledón de la provincia de San José; el cantón de Atenas de la provincia de Alajuela; y el cantón de Aguirre de la provincia de Puntarenas. Al sur con el Océano Pacífico y al oeste con el mismo océano y el Golfo de Nicoya. Por su parte, el clima de la región oscila entre el semiárido del Pacífico Norte y el hiperhúmedo del Pacífico Sur. Esta región posee una extensión territorial de $2835,63 \mathrm{~km}^{2}$ (5,6 \% del territorio costarricense), siendo la más pequeña del país. ${ }^{5}$

Con respecto a la historia de los cantones que hoy componen la Región Pacífico Central costarricense, luego de la independencia en 1825 el país se divide en dos departamentos, los cuales a su vez en distritos y estos en ciudades, villas o pueblos. Para ese año está la Ciudad de Esparza, perteneciente al distrito de Cañas del Departamento Occidental. En 1835 el país se divide en tres departamentos y estos en ciudades, villas, pueblos o poblaciones, para este año pertenecen al Departamento Occidental las poblaciones de Puntarenas y Esparza. ${ }^{6}$

En 1848 la Constitución Política establece cinco provincias (San José, Cartago, Alajuela, Heredia y Guanacaste) y la Comarca de Puntarenas; adherido a la provincia de Alajuela se constituye Esparza como cantón. En 1864 se crea el cantón de San Mateo perteneciente a la provincia alajuelense. En 1883 se agrega Esparza como cantón de la Comarca de Puntarenas. En 1908 es creado el cantón de Orotina, procedente del cantón de San Mateo. En 1915 se establece Puntarenas como provincia y se crea el cantón de Montes de Oro. En 1950 perteneciente a la provincia de Puntarenas se crea el cantón de Aguirre, del cual en 1971 se desprende el cantón de Parrita. En 1980 desprendido del cantón de Puntarenas se establece el cantón de Garabito.?

Como hemos visto, la Región Pacífico Central de Costa Rica es una construcción bastante reciente y no aplica para el periodo que nos compete (1909-1955). Por ende, para efectos de la presente investigación tomaremos la región en su totalidad, de la cual analizaremos su desarrollo agrícola interrelacionándolo con los mercados nacional e internacional. Por otra parte, si 5 Ibid., 28-32

$6 \quad I b i d .$, "División territorial administrativa de Costa Rica: Evolución histórica y situación actual", Serie cantones de Costa Rica 1, Instituto de Fomento y Asesoría Municipal (IFAM), Dirección de Gestión Municipal, Sección de Investigación y Desarrollo, San José, 2003, 11-12

$7 \quad$ Ibid., $13-16$ 
identificamos casos excepcionales o atípicos de un cultivo en un cantón o distrito específico, dicho caso será expuesto.

\section{Agricultura en la Región Pacífico Central}

En este apartado identificamos en primera instancia la variedad de cultivos en la región: café, caña de azúcar, frijoles, maíz, plátanos, bananos, arroz, yuca, papas, tabaco, cacao, caucho, verduras y otros; esto buscando caracterizar el perfil agrícola del Pacífico Central. Para fines analíticos hemos clasificado los cultivos agrícolas en dos tipos: (i) para exportación (café, banano, caña dulce, cacao y el caucho); y (ii) para subsistencia o de consumo interno ${ }^{8}$ (frijoles, maíz, arroz, plátanos, yuca, papas, verduras y diversos cultivos). ${ }^{9}$ Además es examinado el cultivo del tabaco y la evolución del uso del suelo para potreros.

Por otra parte, vale señalar que el identificar diversidad agrícola en la Región Pacífico Central colabora en la tarea ya iniciada de desmitificar las tesis tradicionales de una Costa Rica monocultivista y/o monoexportadora. ${ }^{10}$ Por otra parte, agregamos que la producción de caña y cacao fue tanto para exportación como para subsistencia. El cacao casi no se importó, mientras el azúcar lo hizo cada año a pesar de que la producción nacional tenía la capacidad de abastecer la demanda del país, esto explicado en que en el mercado interno ésta era más cara. ${ }^{11}$

\section{Cuadro 1}

\section{Superficie cultivada en la Región Pacífico Central de Costa Rica (1909-1955)}

$8 \quad$ Excepto en los años de 1915 a 1920 cuando hubo una gran demanda de algunos de ellos en el mercado internacional.

$9 \quad$ Esta tipología agrícola es también utilizada en Emmanuel A. Barrantes Zamora, Hilda María Bonilla Quesada y Olga Marta Ramírez Cortés, "Costa Rica. La disyuntiva agrícola en el periodo 1905-1925: Cultivos de exportación y cultivos de subsistencia”, Anuario de Estudios Centroamericanos 27, n. 2 (2001): 121-161; y William Solórzano Vargas, "Colonización agrícola de la Región Norte de Costa Rica 1884-1955”, Tesis de Maestría en Historia, Universidad de Costa Rica, 2004

10 Sobre éstas véase Rodrigo Facio Brenes, Estudio sobre la económica costarricense, San José: ECR, 1972; Carolyn Hall, El café y el desarrollo histórico - geográfico de Costa Rica, San José: ECR, 1974 y Costa Rica una interpretación geográfica con perspectiva histórica, San José: ECR, 1983; y José Luis Vega Carballo, Hacia una interpretación del desarrollo costarricense: ensayo sociológico, San José: Editorial Porvenir, 1982

11 Barrantes, Bonilla y Ramírez, 125 


\begin{tabular}{|c|c|c|c|c|c|c|c|c|}
\hline \multirow{2}{*}{$\begin{array}{c}\text { Censo } \\
\text { agrícola }\end{array}$} & \multicolumn{2}{|c|}{1909} & \multicolumn{2}{|c|}{1914} & \multicolumn{2}{|c|}{ 1923-1927 } & \multicolumn{2}{|c|}{1955} \\
\hline & ha & $\%$ & ha & $\%$ & ha & $\%$ & ha & $\%$ \\
\hline $\begin{array}{l}\text { Cultivos de } \\
\text { exportación }\end{array}$ & 773,76 & 0,27 & 580 & 0,20 & 1401,14 & 0,49 & 1213,5 & 0,42 \\
\hline $\begin{array}{l}\text { Cultivos para } \\
\text { subsistencia }\end{array}$ & 5340,92 & 1,87 & 5351,5 & 1,87 & 7209,77 & 2,52 & 205902 & 71,95 \\
\hline Potreros & 18738,00 & 6,54 & 16756,00 & 5,86 & 25952,00 & 9,07 & 47752,40 & 16,69 \\
\hline Área cultivada & 24852,68 & 8,68 & 22687,50 & 7,93 & 34562,91 & 12,08 & 254867,90 & 89,06 \\
\hline $\begin{array}{l}\text { Área sin } \\
\text { cultivar }\end{array}$ & 261307,32 & 91,32 & 263472,5 & 92,07 & 251597,09 & 87,2 & 31292,1 & 10,94 \\
\hline
\end{tabular}

*Con respecto al total del área de la región.

Fuentes: Archivo Nacional de Costa Rica (ANCR), Dirección General de Estadísticas y Censos (DGEC), Serie 901, "Cuadros del censo agrícola de la provincia de Alajuela: cantones de Orotina y San Mateo: por cultivos actuales, extensión en manzanas, producto medio por manzana, número de árboles sembrados, beneficios e industrias, industria pecuaria, 1909"; Serie 908, "Cuadros del censo agrícola de la provincia de Puntarenas: cantón central y Esparta: por cultivos actuales, extensión en manzanas, producto medio por manzana, número de árboles sembrados, beneficios e industrias, industria pecuaria, 1909"; Serie 477, Libro de censos agrícolas e industriales, 1923-1927; Serie 16, Censo agrícola de 1955; y Dirección General de Estadística, “Censo Agrícola 1914”, Anuario estadístico año 1913, Tomo decimoséptimo, San José: Tipografía Nacional, 1914

En el Cuadro 1 observamos el desarrollo de la superficie cultivada en la Región Pacífico Central entre los años de 1909 y 1955, así como el del suelo utilizado para potreros y el no utilizado del todo. Este cuadro nos muestra que el uso del suelo para productos de exportación en este periodo no fue relevante, ni siquiera un 0,5\% del área de la región fue explotada para este tipo de cultivos. Por otra parte, encontramos que el uso del suelo en potreros se mantuvo durante los primeros 20 años y prácticamente se duplicó para 1955.

Además, vemos en el Cuadro 1 cómo la agroindustria en la región entre 1909 y 1927 no fue una actividad económica significante, ni siquiera el 3\% del suelo se utilizó para cultivos, gran 
diferencia con respecto a 1955 cuando el territorio utilizado para producción agrícola supera en un $70 \%$ del total de la región. Esto principalmente para productos para subsistencia como lo vemos en el Gráfico 1 y lo veremos en el Cuadro 2.

\section{Gráfico 1}

\section{Desarrollo de tipos de cultivos en la Región Pacífico Central (1909-1955)}

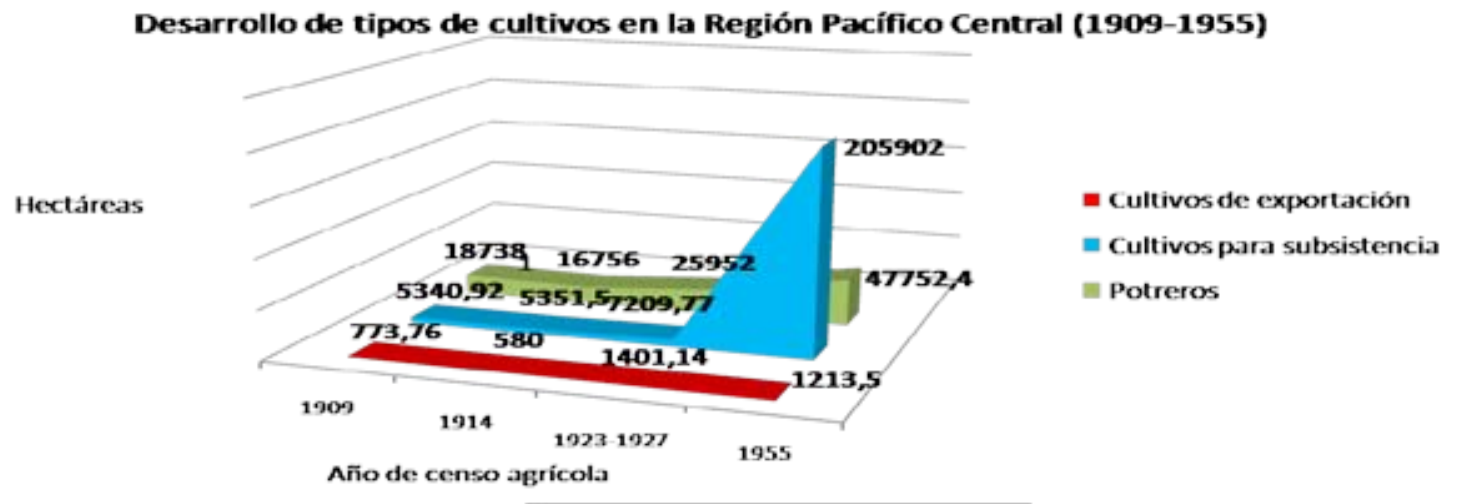

Fuente: La misma del Cuadro 1.

A partir del Gráfico 1 podemos ver el desenvolvimiento del uso del suelo en hectáreas de la región en estudio. Con el Gráfico 1 es más clara la regularidad y el aumento de las zonas destinadas para potreros, así cómo la poca importancia de los cultivos de exportación en la región. También es obvia la incrementación de la producción de cultivos de subsistencia en la Región Pacífico Central para el último año, se pasó de 5340,92 ha cultivadas para dichos productos en 1909 a 205902 ha para 1955. Pero como hemos observado, sucede un cambio importante entre 1927 y 1955, siendo la pregunta: ¿qué sucedió?

La respuesta está en que nos encontramos en una coyuntura mundial de crisis económica. Debemos recordar que desde la independencia Costa Rica optó por un modelo de progreso basado 
en la agroexportación, ${ }^{12}$ por lo que paulatinamente el país experimentó una expansión en lo que respecta a las áreas de cultivo destinadas a productos para exportación, tónica que continúa en la década de $1920 .^{13} \mathrm{El}$ modelo de crecimiento económico costarricense en el primer tercio del siglo XX se sustentaba en la agroexportación.

Con el desarrollo de la I Guerra Mundial y el cierre de los principales mercados europeos, Estados Unidos se convierte en el principal comprador del país. En 1920 el 71\% de las exportaciones son para dicha nación. La guerra trajo también un déficit fiscal debido a la dependencia estatal de los impuestos aduaneros a las importaciones. A partir de ésta el tipo de cambio colón-dólar seguirá aumentando periódicamente al igual que la deuda externa. La guerra creó problemas de crédito en el país. ${ }^{14}$

Entre 1913 y 1928 las exportaciones bananeras declinaron por el auge en Honduras, ${ }^{15}$ pero las del cacao y el café aumentaron, alcanzando el último su auge entre 1926 y 1927, lo cual fue fundamental para la estabilidad económica del país por el declive bananero. Este fue un periodo en que las políticas económicas se subordinaron a las necesidades de la producción cafetalera. ${ }^{16}$ El Estado costarricense optó por una serie de cambios estructurales ${ }^{17}$ en procura de una mayor industrialización y diversificación agrícola no exportadora, sin embargo el desempeño económico siguió dependiendo de la suerte del sector exportador. Para 1928 las exportaciones representaban un 109,6 proporcional del PIB en nuestro país. ${ }^{18}$

12 Iván Molina Jiménez, El legado colonial del modelo agroexportador. Costa Rica (1821-1914), San José: EUCR, 2005

13 Ana María Botey, "Costa Rica entre guerras: 1914-1940”, Serie de Cuadernos de Historia de las Instituciones de Costa Rica (San José) 6 (2005): 57-58

14 Víctor Bulmer-Thomas, La Historia Económica de América Latina desde la Independencia, México: Fondo de Cultura Económica, 1998, 105-228 y Botey, 1-33

15 Ciro Cardoso Ciro y Héctor Pérez, Centro América y la Economía Occidental (1520-1930), San José: ECR, 1977, 275-285; Botey, 58-59, Bulmer-Thomas, 231-272

16 Molina, 33-48

17 Se establecieron rebajas salariales y un sistema de "tercerillas" para los empleados estatales. La inflación y devaluación monetaria fueron una constante, ya que las clases ricas nacionales optaron por guardar su dinero en bancos estadounidenses, lo cual agravó la crisis en los bancos estatales. Entonces se crea un banco estatal, el Banco Internacional, con el propósito de solventar la crisis crediticia y de circulante en los grupos pequeños y medianos de la sociedad. Se puso un impuesto a la exportación del café; se prohibió la exportación de minerales -oro y plata- para así poder aumentar las reservas de éstos; impuestos a las herencias; leyes sobre seguros mercantiles y otros. Recordemos que para la época utilizábamos el patrón oro y que la influencia estadounidense del momento promulgaba el uso de un patrón bimetal; ello porque éstos poseían una de las principales reservas del mundo en plata. Véase: A. G. Kenwood y A. L. Lougheed, Historia del desarrollo económico internacional, Madrid: Istmo, 21ss y Botey, 53-57

18 Bulmer-Thomas, 229-230 
El "boom" de la crisis se da en durante octubre de 1929 con el crack de la Bolsa de Valores de Wall Street en Nueva York. Los precios de los bienes aumentaron cuando la oferta superó a la demanda, se produjeron excesivas demandas de crédito y un alza en las tasas de interés, elevándose el costo de los inventarios y reduciéndose la demanda de productos de postre como los costarricenses (café, banano, caña dulce y cacao específicamente). La fuga de capital fue una presión adicional sobre el país en un momento que éste no poseía capacidad de respuesta.

Para 1929 el mercado internacional colapsa con una Costa Rica dependiente de éste, a pesar de esto las exportaciones cayeron sólo en un 25\%, poco en comparación a otros países latinoamericanos. ${ }^{19}$ Pronto iniciaría la II Guerra Mundial con lo que el mercado internacional agravaría la crisis, por lo que el Estado costarricense se esfuerza por expandir sus fronteras agrícolas en procura de aumentar la producción de cultivos para la subsistencia.

En el Cuadro 2 observamos la diversidad de cultivos en la región, la cual se mantuvo entre 1909 y 1955. En primera instancia vemos como ningún cultivo de exportación fue significante durante el periodo. Señalamos que en San Mateo el cultivo del café disminuyó de 83,3 ha a 36,2 ha cultivadas entre 1909 y el periodo 1923-1927, lo cual ha sido explicado en razón a un aumento demográfico que desplazó la producción cafetalera. ${ }^{20}$ Pero consideramos más bien que se debió a que el cantón buscó especializarse en el cultivo del arroz, de 144,9 ha cultivadas en 1909 pasó a 316,8 en el periodo 1923-1927 y a 14094,2 ha en 1955 (en este último año además el cultivo del café se incrementó a 188,6 ha cultivadas).

19 Bulmer-Thomas, La economía política de Centroamérica desde 1920, San José: BCIE, 1989, 113-172 y La Historia Económica de América Latina desde la Independencia, 231-237

20 Barrantes, Bonilla y Ramírez, 132 


\section{Cuadro 2}

\section{Diversidad de cultivos en la Región Pacífico Central de Costa Rica (1909-1955)}

1909

1914

Ha

Producto

$\begin{array}{ccccccc}\text { Ha } & \% & \text { Ha } & \% & \begin{array}{c}\text { Ha } \\ \text { cultivadas }\end{array} & \% & \begin{array}{c}\text { Ha } \\ \text { cultivadas }\end{array}\end{array}$

Cultivos de exportación

$\begin{array}{lcccccccc}\text { Café } & 212,27 & 0,85 & 160,00 & 0,71 & 251,20 & 0,73 & 605,00 & 0,24 \\ \text { Banano } & 26,00 & 0,10 & 27,50 & 0,12 & 508,39 & 1,47 & 137,70 & 0,05 \\ \text { Caucho } & 53,90 & 0,22 & 18,50 & 0,08 & 47,00 & 0,14 & - & - \\ \text { Cacao } & 34,99 & 0,14 & 31,00 & 0,14 & 150,95 & 0,44 & - & - \\ \text { Caña dulce } & 446,60 & 1,80 & 343,00 & 1,51 & 443,60 & 1,28 & 470,80 & 0,18\end{array}$

Cultivos para subsistencia

\begin{tabular}{|c|c|c|c|c|c|c|c|c|}
\hline Frijoles & 649,10 & 2,61 & 346,00 & 1,53 & 890,40 & 2,58 & 49 358,30 & 19,37 \\
\hline Maíz & 2 458,20 & 9,89 & 3 212,00 & 14,16 & 2549,60 & 7,38 & 10447,00 & 4,10 \\
\hline Arroz & 1079,90 & 4,35 & 814,00 & 3,59 & 1387,20 & 4,01 & 48 850,90 & 19,17 \\
\hline Plátanos & 920,80 & 3,71 & 855,00 & 3,77 & 877,00 & 2,54 & 484,50 & 0,19 \\
\hline Yuca & 84,00 & 0,34 & 99,00 & 0,44 & 607,00 & 1,76 & 7 775,20 & 3,05 \\
\hline Papa & - & & 4,00 & 0,02 & 217,40 & 0,63 & 15,70 & 0,01 \\
\hline Verduras & 40,60 & 0,16 & - & - & 218,25 & 0,63 & 3474,30 & 1,36 \\
\hline Diversos cultivos & 76,65 & 0,31 & - & - & 393,27 & 1,14 & 85457,60 & 33,53 \\
\hline Tabaco & 31,67 & 0,13 & 21,50 & 0,09 & 69,65 & 0,20 & 38,50 & 0,02 \\
\hline Potreros & 18738,00 & 75,40 & 16756,00 & 73,86 & 25952,00 & 75,09 & 47752,40 & 18,74 \\
\hline Total & 24852,68 & 100 & 22 687,50 & 100 & 34 562,91 & 100 & 254867,90 & 100 \\
\hline
\end{tabular}

*Este valor es con respecto al total de la superficie cultivada

Fuente: La misma del Cuadro 1. 
Por su parte, el cultivo de exportación más importante en la Región Pacífico Central: la caña de azúcar, tan sólo fue producido en más de 1\% de las áreas cultivadas en cada año. Entre 1909-1914 el área cultivada de caña disminuye en los cantones de San Mateo, Puntarenas, y Esparza debido a la baja en la demanda del producto por la elevación de los costos de transporte y la especialización de este cultivo en Grecia (que incluía a Poás y parte de Naranjo) en Alajuela, Jiménez-Turrialba en Cartago y Liberia en Guanacaste. ${ }^{21}$

Por otro lado, con respecto al banano el cantón de Puntarenas tuvo un crecimiento importante pues de menos de 50 ha en 1914 llegó a tener más de 400 ha en 1925. Por su lado, Esparza de las pocas hectáreas que destinaba al cacao terminó dejándolo de producir, esto debido a que la provincia de Limón se empezó a especializar en la producción de este cultivo de exportación. ${ }^{22}$ Los datos anteriores nos hacen pensar que la producción de los cultivos de exportación se debió tan sólo para el consumo interno de los pobladores de la región, conclusión similar a la que llegan Emmanuel Barrantes, Hilda Bonilla y Olga Ramírez sobre el café y el banano. ${ }^{23}$

Por otra parte, podemos constatar que el cultivo de arroz y frijoles cumplió un papel preponderante en el incremento de los cultivos de subsistencia en la región, de un 7\% del terreno destinado para estos se pasó a un 40\%. En este aumento marcó diferencia el cantón de Puntarenas que de 850 ha en 1909 (incluye Montes de Oro, Aguirre (Parrita) y Garabito), en 1955 (ya no incluye Montes de Oro) cultivó más de 62500. También resalta el cantón de Esparza que de 200,9 ha cultivadas en 1909 pasa a 12627,3 ha en 1955. Este aumento lo explicamos en el hecho de que en los cantones de San Ramón, Grecia y Poás se redujo la producción de frijoles para especializarse al menos en los dos últimos cantones en la caña dulce. ${ }^{24}$

Por otro lado, el cultivo del maíz durante los primeros veinte años fue el producto más importante, siendo también Puntarenas el cantón que permitiera ello, de las 2458,2 ha cultivadas en el primer año 1338,9 ha eran de este cantón, de las 3212 ha en 19141337 ha y de las 2549,6

21 Hugo Guzmán y Jaime Murillo, "Evolución de la industria de la caña de azúcar en Costa Rica: 19151963”, San José: CSUCA, 1978

22 Juan Rafael Quesada Camacho, "Algunos aspectos de la historia económica del cacao en Costa Rica: 1880-1930”, Revista de Historia (Heredia) 5 (1977): 65-100 y “Comercialización y movimiento coyuntural del cacao", Revista de Historia (Heredia) 6 (1978): 69-110

23 Barrantes, Bonilla y Ramírez, 132-133

24 Ibid., 136 
del periodo 1923-1927 (sin Montes de Oro) 1391,4 ha. Para los años en estudio, sin duda alguna Puntarenas era de los graneros más importantes del país, ${ }^{25}$ su producción en granos aumentó a pesar de la fragmentación que vivió durante el periodo en estudio.

También el Cuadro 2 muestra que el cambio más significante está en "diversos cultivos", que en 1909 significaba 0,31\% del área cultivada, en el periodo 1923-1927 el 1,14\% y para en 1955 el 33,53\%. Entre "diversos cultivos" poseemos datos específicos sobre cuáles son hasta 1955, el censo habla de guineo, ajonjolí, maní, ajos, cebolla, repollo, tomate, chile dulce y zanahoria. Sobre los cultivos anteriores sobresale San Mateo y Orotina, con 42464,9 ha y 40758,2 ha respectivamente, destinadas al cultivo del guineo, es decir el 97,4\% del área correspondiente a "diversos cultivos". Finalmente, observamos la supremacía -ya señalada- de la superficie destinada para potreros en los tres primeros censos y resaltemos el hecho de que si bien en pocas hectáreas, el tabaco siempre se cultivó y de paso en todos los cantones de la región.

\section{Conclusión}

La Región Pacífico Central de Costa Rica posee una extensión territorial de 2835,63 km² y la compone los cantones Puntarenas, Esparza, Montes de Oro, Garabito, San Mateo y Orotina. La agricultura en esta región fue bastante variada entre 1909 y 1955, encontramos plantaciones de cultivos agrícolas para exportación (café, banano, caña dulce, cacao y el caucho) y para subsistencia (frijoles, maíz, arroz, plátanos, yuca, papas, verduras y diversos cultivos). También es cultivado tabaco y se destinan áreas para potreros.

El uso del suelo en la región para productos de exportación no fue relevante, ni siquiera un $0,5 \%$ del área fue explotada para este tipo de cultivos. En lo que respecta al área destinada a potreros se mantuvo durante los primeros 20 años y prácticamente se duplicó en 1955. La agroindustria en la región entre 1909 y 1927 no fue una actividad económica significante (3\% del territorio para cultivos), pero 1955 el territorio utilizado para producción agrícola supera en un 70\% del total de la región. La producción de cultivos de subsistencia pasó de 5340,92 ha cultivadas en 1909 a 205902 ha en 1955.

La coyuntura de crisis económica mundial de la primera mitad del siglo XX lleva al 
Estado costarricense a realizar importantes cambios estructurales. De un modelo de crecimiento económico agroexportador dependiente del mercado internacional, se promueve un modelo de industrialización y diversificación agrícola no exportadora. La diversificación agrícola promovió la colonización de nuevas áreas para cultivos de sustento nacional, o enfocados al mercado interno, lo cual sucedió en la Región Pacífico Central.

La diversidad de cultivos en la región se mantuvo entre 1909 y 1955. Ningún cultivo de exportación fue significante durante el periodo. El cultivo de exportación más importante fue la caña de azúcar, pero entre 1905 y 1914 el área cultivada disminuye en los cantones de San Mateo, Puntarenas, y Esparza (además en este cantón se dejó de producir cacao), todo debido a la especialización en otros lugares. El café disminuyó de 83,3 ha a 36,2 ha cultivadas entre 1909 y el periodo 1923-1927 en San Mateo. El banano creció en Puntarenas de 50 ha en 1914 a más de 400 ha en 1925. Consideramos que la producción de los cultivos de exportación se debió para el consumo interno de los pobladores de la región.

Por otra parte, el cultivo de granos (arroz, frijoles y maíz) cumplió un papel preponderante en el incremento de los cultivos de subsistencia en la región, de un 7\% del terreno destinado para estos se pasó a más de un 40\%. En este aumento marcó diferencia Puntarenas que de 850 ha en 1909 cultivó más de 65000 en 1955. Por otro lado, sobresalen con 42464,9 ha y 40758,2 ha destinadas al cultivo del guineo (el 97,4\% del área correspondiente a "diversos cultivos"), San Mateo y Orotina en 1955 respectivamente. 